\title{
Virtual Humans for Assisted Health Care
}

\author{
Patrick Kenny, Thomas Parsons, Jonathan Gratch, Albert Rizzo \\ University of Southern California's Institute for Creative Technologies \\ 13274 Fiji Way \\ Marina del Rey, CA 90292 \\ United States of America \\ $\{$ kenny, tparsons, gratch, rizzo $\} @$ ict.usc.edu
}

\begin{abstract}
There is a growing need for applications that can dynamically interact with aging populations to gather information, monitor their health care, provide information, or even act as companions. Virtual human agents or virtual characters offer a technology that can enable human users to overcome the confusing interfaces found in current human-computer interactions. These artificially intelligent virtual characters have speech recognition, natural language and vision that will allow human users to interact with their computers in a more natural way. Additionally, sensors may be used to monitor the environment for specific behaviors that can be fused into a virtual human system. As a result, the virtual human may respond to a patient or elderly person in a manner that will have a powerful affect on their living situation. This paper will describe the virtual human technology developed and some current applications that apply the technology to virtual patients for mental health diagnosis and clinician training. Additionally the paper will discuss possible ways in which the virtual humans may be utilized for assisted health care and for the integration of multi-modal input to enhance the virtual human system.
\end{abstract}

\section{Categories and Subject Descriptors}

I.2.11 [Distributed Artificial Intelligence]: Intelligent Agents and Multiagent Systems

\section{General Terms}

Advanced 3D modeling and animation technology. Applications, experience reports and user studies. Models of personality, emotion, and social behavior. Multi-user/multi-virtual-agent interaction. Verbal and non-verbal expressiveness. Experimentation, Algorithms, Design, Human Factors.

\section{Keywords}

Virtual Humans, Virtual Patients, Health Care, Game Characters

Permission to make digital or hard copies of all or part of this work for personal or classroom use is granted without fee provided that copies are not made or distributed for profit or commercial advantage and that copies bear this notice and the full citation on the first page. To copy otherwise, or republish, to post on servers or to redistribute to lists, requires prior specific permission and/or a fee.

PETRA 2008, July 15-19, 2008, Athens, Greece.

Copyright 2008 ACM XXXXXXXXX...\$5.00.

\section{INTRODUCTION}

As people age, there is a growing interest in maintaining good health, active social participation, and independent living. An older aged person's condition, however, may require one to stay in their homes and may not allow these interests to be realized. This in turn increases the stress upon caregivers. Whilst there is a growing need to monitor and care for the aging population there is also a need to reduce the cost and burden. The use of technology to help with these issues is readily apparent. Hence, there is an ever increasing need to develop novel technologies that can monitor patient status, track patient behavior or health needs, and substitute for health care professionals. Such technologies can enhance the accomplishment of everyday tasks (e.g. medication adherence reminders, answer questions, automated data gathering, and report delivery to primary care physicians). Current computer interfaces are cumbersome for patients to use, especially elderly persons that are not adept at using complex software. What is required is a human like and natural interaction with the computer. Herein we present a novel approach that uses embodied conversational virtual human characters that can monitor the older aged persons, record data from sensors, and communicate that data to health care professionals.

Virtual Human Agent technology has evolved to a point where researchers are developing characters in virtual reality applications that can act as virtual patients for interpersonal skills training, agents that can engage trainees in cultural bi-lateral negotiation training or just act as interactive characters for entertainment or promotional purposes. These virtual human agents have the ability to recognize speech, respond to questions and generate verbal and non-verbal behavior.

This paper describes current research in building virtual humans and virtual patient applications and addresses some areas where these can be applied to health care assistance for both in-home and hospitalized patient care. The work presented here is a preliminary attempt at what we believe to be a large application area that can be applied towards assisted health care.

\section{RELATED WORK}

There have been a small, but growing number of efforts to develop integrated virtual humans. These have been applied to a variety of applications including training, tutoring, marketing and entertainment. Some current research efforts include the work of Justine Cassell's group at Northwestern [4], Tim Bickmore's work on relational agents for health [2], Elisabeth André at the 
University of Augsburg [11] and Jonathan Gratch's group at University of Southern California $[6,8,12]$. Considerable work is also being conduced on performing studies to better understand the kinds of behavior humans perform while conversing with humans or other agents, including conversation, rapport and nonverbal gestures using speech and vision sensor systems $[7,10]$.

There have also been several efforts to utilize virtual humans for medical applications. For example, Benjamin Lok's group at the University of Florida has performed research with virtual patients for medical examination and training [1]. Instead of exclusively relying on the costly and labor intensive approach of hiring professional "standardized" patients for novice medical students to practice on, they constructed a virtual environment to represent an examination room where a virtual patient could be interviewed verbally or 'physically' examined. There has also been considerable work on building socially interactive robots that exhibit human like qualities, Cynthia Breazeal [3], Maja Mataric [9] or to help patients deal with disabilities [13].

\section{VIRTUAL HUMANS}

Virtual humans are developing into powerful interfaces that can enable greatly increased intuitive human like interactions. These virtual human systems consist of characters that have realistic appearances, can think and act like humans, and can express themselves both verbally and non-verbally. Additionally, these virtual humans can listen and understand natural language and see or track limited user interactions with speech or vision systems. While our work has concentrated on creating realistic characters, there are many examples of cartoon like characters in computer video games and movies or even robotic animals and dinosaurs that give comfort to kids and the elderly alike. Our work is differentiation from these characters in that our virtual humans offer an interactive experience. Further, our virtual humans are capable of listening to users, reasoning about the situation at hand based on sensor input and the situational context and choosing appropriate dialog and actions and delivering those actions in generated verbal output that is synchronized to realistic human like non-verbal animations.

Building virtual humans requires fundamental advances in $\mathrm{AI}$, speech recognition, natural language understanding and generation, dialog management, cognitive modeling and reasoning, virtual human architectures and computer graphics and animations. All of these technologies need to be integrated together into a single system that can work in unison, be expandable, flexible and plug-and-play with different components.

The virtual human systems we develop include complex cognitive agents, question response agents, and culturally specific characters. These virtual characters have beliefs, desires and intentions, as well as daily routines that can be carried out within virtual worlds. These agents are based around a set of distributed components that communicate with each other and perform specific tasks (e.g. speech recognition). Although these agents do require time and effort for adequate development, we have tried to address this with a distributed underlying virtual human architecture. Given the distributed nature of the architecture, we are able to replace components without large integration efforts, which reduces the time needed to build or expand a given application. Additionally a set of tools is continually being developed to ease the task of creating these characters. The major components in the system, as seen in Figure 1 are:

- Speech Recognition: digitizes the user's speech and produces a string of words as output.

- Natural Language Understanding and Dialogue Management: parses the word string produced by speech recognition and extracts meaning and concepts to form an internal semantic representation.

- Intelligent Agent: reasons about plans and generates actions based on its internal state and the input from the verbal text or other multi-modal devices. Complex agents can be created using a cognitive architecture that reason about plans and actions and integrate a dialog manager and emotion model. Simple agents can be created with a question response system that picks a response based on statistical analysis of the input text. The output of the agent is a response output string and actions.

- Non-Verbal Behavior Generation: takes the response output string and applies a set of rules to select gestures, postures and gazes for the virtual characters.

- Procedural Animation System: synchronizes speech output with gestures and other non-verbal behavior to control the characters in the virtual environment.

- Speech Generation: performs speech synthesis from the text generated by the agent. Or alternatively a set of pre-recorded voice strings can be used as the speech.

- Game Engine Graphic Display: is the current underlying graphics engine used to display the character and virtual environment.

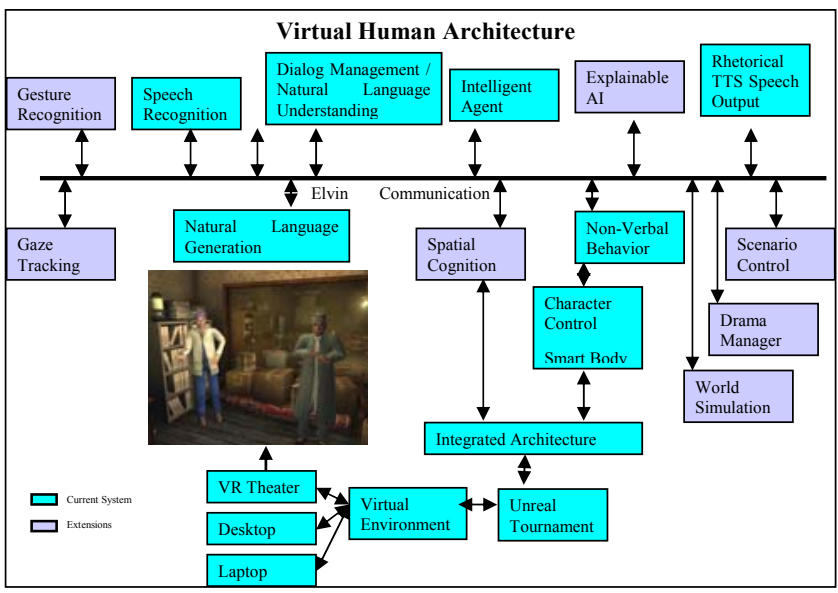

Figure 1. Virtual Human Architecture Components

\subsection{Virtual Human Example Scenario}

To provide proof of concept a scenario was constructed to demonstrate the research and virtual human architecture integration. The scenario consisted of a Stability and Support Operations scenario in which a user interacts with two virtual humans: a Spanish doctor, head of a Non-Government Organization clinic, and an Iraqi village elder. Set in a small Iraqi town plagued by violence, the human trainee takes on the role of an Army captain with orders to move the clinic to a safer location. It is up to the user to negotiate this goal with both the doctor and the elder in order to reach agreement between all stakeholders. This scenario displays various virtual human research features, including multi-party negotiation, dynamically generated non- 
verbal behavior and a central knowledge base ontology. Figure 2 is an example picture of the scenario.

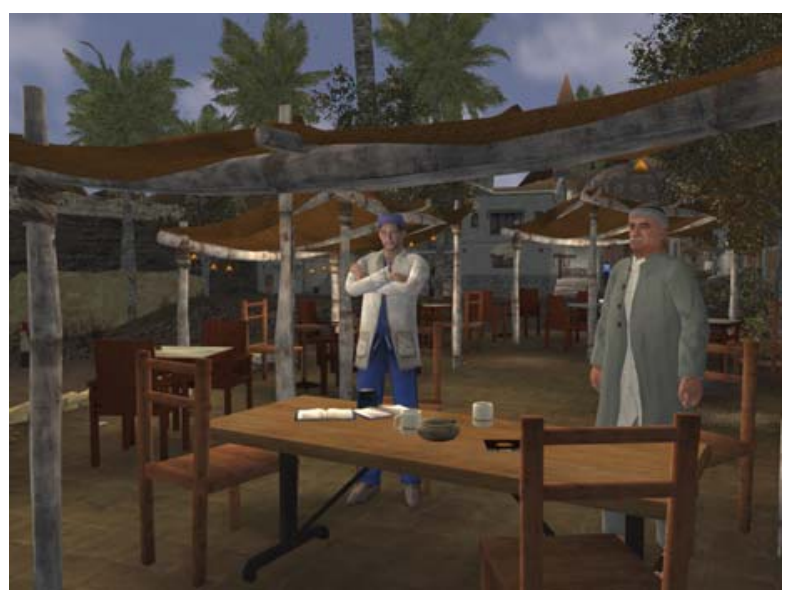

Figure 2. Negotiation Scenario

\section{VIRTUAL PATIENTS}

Extending virtual humans to health care has been accomplished with virtual patients (VP). VPs are virtual interactive agents designed to simulate a particular clinical presentation of a patient with a high degree of consistency and realism. VPs have recently been implemented to teach bedside competencies of bioethics, basic patient communication and history taking, clinical decision making, and psychotherapy training. These VPs have been found to provide valid, reliable, and applicable representations of live patients; and can augment many live actor simulated patient programs. VPs could also be used to teach caregivers or family members how to communicate or interact with their aging loved ones or relatives with mental health issues. For example, a VP could simulate an elderly person requiring a great deal of facilitation with their activities or mental states of daily living, and the caregiver would be required to attend to these needs. The concept is to create virtual characters that have a set of human like characteristics, such as beliefs, desires, goals and intentions, and psychological problems that can be exhibited in the character for the desired type of training or education scenario.

\subsection{Virtual Patients for Clinician Training}

We have successfully applied our virtual human technology to the creation of virtual patients for teaching novice clinicians differential diagnostic skills [8]. The first prototype was a character called 'Justin', See Figure 3. Justin is a teenage boy that exhibited a conduct disorder condition as described in the DSMIV mental health manual [5]. The user was tasked to asses the condition of the patient and get a preliminary history. The users interacted with the character (using speech recognition) and the system generated verbal and non-verbal responses based upon the mental health condition portrayed by the virtual patient. The evaluation of the system was two fold: 1 ) assess the underlying technology of the system as a whole for its interaction ability and capability to display an engaging character; and 2) assess if it could be used to train a clinician's interview and diagnosis skills. Subject testing of the system was conducted with students and actual clinicians. The data was collected through pre and post questionnaires and $\log$ files collected during the interview. Preliminary results were positive as most users felt engaged and asked a fair amount of questions to assess the patients' history and condition. The task of building these characters for different conditions requires a considerable amount of time and desperately needs more advanced toolsets.

\subsection{Virtual Humans as Health Care Assistants}

Virtual humans can also be developed to function as health care assistants. They could be used as characters that users interact with that portray simulated doctors or as training or education tools. They can represent human like interfaces to the vast knowledge of the web for users with disabilities to ask questions, or more specifically they could ask questions about their own health issues. They could monitor the environment through a set of sensors and act like health care professionals to remind patients of their health needs, for example reporting the last time they took their medicine or performed their physical therapy. They could also act as companions for the aging or home ridden patients. To be more effective the virtual human systems would be extended to gather and store multi-modal data about the patient and the environment to better reason about the patients' condition and needs.

\subsection{Multi-Modal Input}

Integration or embedding of sensors or cameras into objects and the home environment will greatly assist the virtual human system. The data from the embedded sensors can be used to record the actions of the user, to keep a history profile of a patient, for example to observe abnormal behavior patterns. The system can monitor emergency situations, for example if a person falls down then the system can automatically call for help or try to communicate with the person to assess their condition. A virtual human like interface would provide a powerful accessible way for people to interact with the underlying technology. Additionally the characters can be customized with personality profiles or genders to better match with the patient. They could have a nice bed-side manner and always be attentive and calm.

\subsection{Large Systems Integration}

Systems like these can be very powerful; however they are not without their own complexity. They need to be reliable and they need to be flexible. Virtual humans can provide basic interactive assistant characters with a single computer or they can be developed to provide a wide range of monitoring and reasoning with many interconnected sensors and components all hooked up to the internet. It will require a multidisciplinary approach that includes researchers from electronics sensors, computer science to psychology and health care administration. A set of common interfaces should be developed for components to communicate and provide specific information. Lastly there needs to be pilot data on what works and how to validate the actions of the virtual humans and systems. 


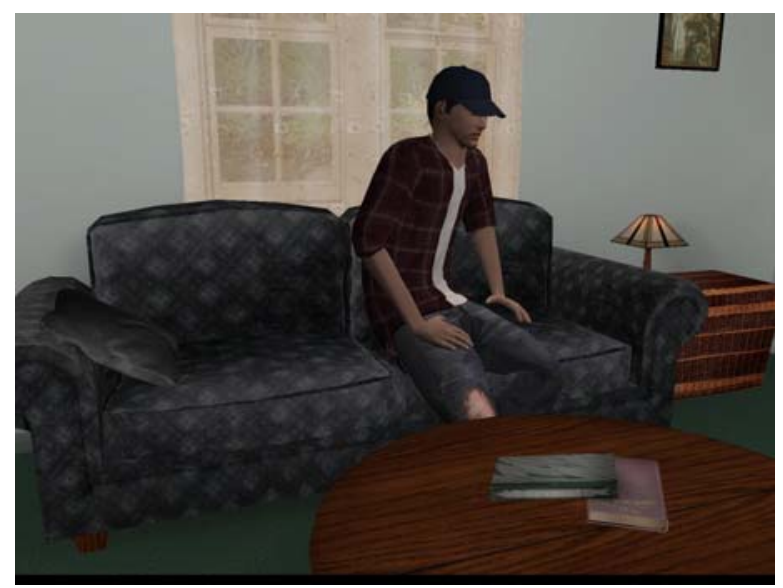

Figure 3. Virtual Patient "Justin" in the clinician's office

\section{FUTURE WORK}

The assisted health care area is an exciting venue for the application of living virtual humans capable of monitoring and reasoning about a patient's health needs and communicating that data through a natural interface.

As we continue to build virtual humans and integrate more multimodal sensor input we will increase the capabilities of these systems. If they are built in a distributed manner, they will be flexible and expandable. Although there are still many research areas and hard problems to be resolved, the needs of an ever increasing aging population will be aided by the inclusion of novel technologies. The community should layout a research plan for the areas that would enhance the integration of virtual humans, sensors and health care for these environments. Agreements upon appropriate standards for interfaces to sensors and systems will allow research areas to build on one another.

Virtual humans are powerful casual interfaces with affective and cognitive reasoning systems behind them, they can affectively respond and reason about the input text or vision they receive and they can produce both verbal and nonverbal output. They can also keep track of large set of data through tracking systems and then reason over that corpus of data to assist the end user. There is potential that they will also have understandings about how to care for aging patients and/or persons with various mental or physical health problems and recommended solutions for the endusers. Further there is the possibility that they could be used by the health professional to get information about the daily activities of patients and can be queried by the health care provided about specific issues with the patient. Ultimately this technology could be in the future households of all patients that need assisted health care.

\section{ACKNOWLEDGMENTS}

This work was sponsored by the U.S. Army Research, Development, and Engineering Command (RDECOM), and the content does not necessarily reflect the position or the policy of the Government, and no official endorsement should be inferred.

\section{REFERENCES}

[1] Andrew, R., Johnsen, K., Dickerson, R., Lok, B,. Cohen, M., Stevens, A., Bernard, T., Oxendine, C., Wagner, P., Lind, S.: Comparing Interpersonal Interactions with a Virtual Human to those with a Real Human, IEEE Transactions on Visualization and Computer Graphics.(2006)

[2] Bickmore, T., Pfeifer, L., and Paasche-Orlow, M. (2007) Health Document Explanation by Virtual Agents Intelligent Virtual Agents '07, Paris.

[3] Breazeal C. 2002. Designing Sociable Robots, MIT Press.

[4] Cassell, J., Bickmore, T., Billinghurst, M., Campbell, L., Chang, K., Vilhjálmsson, H., and Yan, H.: "An Architecture for Embodied Conversational Characters.", Proceedings of the First Workshop on Embodied Conversational Characters, October 12-15, Tahoe City, California. (1998).

[5] Diagnostic and Statistical Manual of Mental Disorders DSMIV-TR $4^{\text {th }}$ Ed, (Text Revision), American, Psychiatric Association, (1994)

[6] Gratch, J., Rickel, J., André, E., Badler, N., Cassell, J., Petajan, E.: Creating Interactive Virtual Humans: Some Assembly Required, IEEE Intelligent Systems, July/August, 54-63, (2002)

[7] Jonathan Gratch, Anya Okhmatovskaia, Francois Lamothe, Stacy Marsella, Mathieu Morales, R. J. van der Werf and Louis-Philippe Morency. Virtual Rapport. 6th International Conference on Intelligent Virtual Agents, Marina del Rey, CA, 2006

[8] Kenny, P., Parsons, T., Gratch J., Leuski, A., Rizzo A.: Virtual Patients for Clinical Therapist Skills Training. 7th International Conference on Intelligent Virtual Agents, pp 197-210, Paris France. (2007).

[9] Maja J Matarić, "Socially Assistive Robotics", IEEE Intelligent Systems, 21(4), Jul/Aug 2006, 81-83

[10] Nevatia, R. Hongeng, S. and F. Bremond, F. Nov 2004. "Video-based event recognition: activity representation and probabilistic recognition methods", CVIU, Vol. 96, No. 2, pp. $129-162$

[11] Rist, Thomas, Andre, Elisabeth and Baldes, Stephan (2003): A flexible platform for building applications with life-like characters. In: Johnson, Lewis and Andre, Elisabeth (eds.) International Conference on Intelligent User Interfaces 2003 January 12-15, 2003, Miami, Florida, USA. pp. 158-165

[12] Swartout, W., Gratch, J., Hill, R., Hovy, E., Marsella, S., Rickel J., Traum, D.:Toward Virtual Humans, AI Magazine, v.27(1). (2006)

[13] Tamura, Toshiyo, Satomi Yonemitsu, Akiko Itoh, Daisuke Oikawa, Akiko Kawakami, Yuji Higashi, Toshito Fujimooto, and Kazuki Nakajima. "Is an Entertainment Robot Useful in the Care of Elderly People With Severe Dementia?" The Journal of Gerentology 59A (January 2004): 83-85. 